\title{
Comparison of EMG during passive stretching and shortening phases of each muscle for the investigation of parkinsonian rigidity
}

\author{
Yuri Kwon ${ }^{\mathrm{a}}$, Ji-Won Kimª , Ji-Sun Kim ${ }^{\mathrm{a}}$, Seong-Beom Koh ${ }^{\mathrm{b}}$, Gwang-Moon Eom ${ }^{\mathrm{a}, \mathrm{c}, *}$ and Tae- \\ Hong Lim ${ }^{\mathrm{d}}$ \\ ${ }^{a}$ School of Biomedical Engineering, Konkuk University, Choonju, 380-701, Korea \\ ${ }^{b}$ Department of Neurology, Korea University College of Medicine, Seoul, Korea \\ ${ }^{c}$ Research Institute of Biomedical Engineering, Konkuk University, Choonju, 380-701, Korea \\ ${ }^{d}$ Department of Biomedical Engineering, University of Iowa, IA, USA
}

\begin{abstract}
The aim of this study was to test the hypothesis in the literature that torque resistance of parkinsonian rigidity is the difference between the independent contributions of stretched and shortened muscles. The hypothesis was tested using muscle-specific stretch-shortening (MSSS) EMG ratio in this study. Nineteen patients with idiopathic Parkinson's disease (PD) and 18 healthy subjects (the mean age comparable to that of patients) participated in this study. The EMG activity was measured in the four muscles involved in wrist joint movement, i.e. flexor carpi radialis, flexor carpi ulnaris, extensor carpi radialis and extensor carpi ulnaris. The passive flexion-extension movement with a range of $\pm 30^{\circ}$ was applied at wrist joint. Root mean squared (RMS) mean was calculated from the envelope of the EMG for each of stretching and shortening phases. MSSS EMG ratio was defined as the ratio of RMS EMG of stretching phase and RMS EMG of shortening phase of a single muscle, and it was calculated for each muscle. MSSS EMG ratios were smaller than one in all muscles. These results indicate that all wrist muscles generate greater mean EMG during shortening than during stretching. Therefore, the torque resistance of parkinsonian rigidity cannot be explained as the simple summation of independent antagonistic torque pair.
\end{abstract}

Keywords: EMG, parkinsonian rigidity, passive stretching, passive shortening

\section{Introduction}

Rigidity is one of the cardinal features of Parkinson's disease and it is defined as the increased resistance during passive joint movement [1]. Understanding the mechanism of rigidity is important for the effective treatment of rigidity [2]. Therefore, many researchers have tried to investigate the mechanism of rigidity [2-9].

Some of them suggested that rigidity is caused by the increased stretch reflex [3-5]. Stretch reflex is

\footnotetext{
* Address for correspondence: Gwang-Moon Eom, School of Biomedical Engineering, Konkuk University, Choonju, 380 701, Korea. Tel.: +82-43-840-3764; Fax:+82-43-851-0620; E-mail: gmeom@kku.ac.kr.
}

0959-2989/15/\$35.00 @ 2015 - IOS Press and the authors. 
the muscle contraction in response to the passive muscle lengthening. The muscle force generated by stretch reflex is to work in the opposite direction to the imposed movement, which resists the movement [2]. On the other hand, rigidity was also suggested to be related to the enhanced shortening reaction [6-9]. Shortening reaction indicates the muscle contraction in response to the passive shortening. Then, the generated muscle force would work in the same direction of the imposed movement, which assists the movement [2].

$\mathrm{Xia}$ et al. suggested that the rigidity is caused by the interaction of stretch reflex and the shortening reaction [2]. They suggested that the resultant torque resistance during passive movement is a summation of the contributions of stretched muscles minus the contributions of shortened muscles, where stretched and shortened muscles are antagonistic each other. Their suggestion was based on the implicit assumption that the antagonistic muscles would generate torques independently and in opposite directions. Specifically, they defined the EMG ratio as the normalized EMG of stretched muscle divided by the normalized EMG of shortened muscle and the normalization was done with the amplitude of resting state EMG. The EMG ratio showed a tendency to increase with mechanical work, called as the objective rigidity score in this paper $(\mathrm{r}=0.212$ to 0.792$)$. They argued these results support their assumption that the net torque (sum of independent torques) induces rigidity.

However, their results have following limitations. First, the correlation between the EMG ratio and the mechanical work was significant only in some experimental conditions (five cases out of eight), and the correlation coefficients were only low to moderate ( $\mathrm{r}=0.438$ to 0.792$)$. Moreover, the mechanical work regarded utilized in their work could not reflect the clinical evaluation of rigidity (rigidity scores in the UPDRS) well (Spearman's rho $=-0.248$ to +0.578 ). That is, the validity of the relationship between the EMG ratio and the severity of rigidity is low, because both relationships of the EMG ratio-mechanical work and the mechanical work-clinical rigidity score were had only low to moderate significance.

There is also fundamental methodological weakness that the EMG during movement cannot be compared either among different muscles or in the identical muscle, because muscle force is well known to depend on the muscle length and velocity. Moreover, not fully validated is Xia et al.'s implicit assumption that the torque from stretched muscle and the torque from shortened muscle are independent each other and the muscle torque resistance is the summation of these active torques. There still remains the possibility that antagonistic muscle generated active torques are not independent. Rather, it would be more feasible that the torque resistance comes from the transient alteration of passive mechanical properties due to antagonistic muscle contractions, because the rigidity itself is not an active property but is defined as passive resistance, i.e., the resistance occurs only when the joint is forced to move.

In spite of all above limitations and objections, if the suggestion and assumption of Xia et al. is valid, then one muscle should show more muscle activity during passive stretch than during passive shortening. Xia et al. derived EMG ratio from antagonistic muscle pairs with normalization by resting state EMG, which led to ambiguous interpretation of the EMG ratio, i.e., its magnitude cannot be translated into resistance or assistance. If we derive EMG ratio from a single muscle (EMG during stretch divided by EMG during shortening), it renders more direct interpretation of the ratio into overall resistance or assistance irrespective of the normalization method. If the ratio is greater than one, it would indicate the muscle is resisting more than assisting the applied passive movement. Otherwise, the muscle is more assisting the movement. We would label this as the muscle specific stretchshortening EMG ratio (MSSS EMG ratio) to distinguish from the EMG ratio suggested by Xia et al..

Therefore, the purpose of this study was to investigate the validity of suggestion of Xia et al. by using MSSS EMG ratio, which could lead to better understanding of the mechanism of rigidity. 


\section{Method}

\subsection{Subjects}

Nineteen patients (age: 69.1 \pm 9.6 yrs, nine males, ten females) with idiopathic Parkinson's disease (PD) and 18 healthy subjects with the mean age comparable to that of patients (age: $67.7 \pm 10.9 \mathrm{yrs}$, seven males, eleven females) participated in this study. All subjects received full explanation of this study before the recruitment. All subjects gave written informed consents. This study was approved by the institutional review board of ethics committee in Korea University hospital.

Table 1 shows the characteristics of patients. A neurologist rated the rigidity score of the wrist in each patient based on the unified Parkinson's disease rating scale (UPDRS) before the experiment.

\subsection{Experimental setup \& procedures}

Figure 1 shows motorized device that applies passive movement for wrist joint. A servo motor (APM-SA01ACN, AC servo motor, Korea) mounted beside the arm-plate applied isokinetic wrist movement. Wrist joint angle was measured by an encoder in the servomotor. Details of the experimental setup is documented in the previous study [10].

Subjects sat on a comfortable chair with their arm on the motorized device. The wrist joint was aligned to the rotation axis of the hand plate. Forearm is fixed on arm plate by strap to prohibit arm movement such as pronation and supination. Subjects were instructed to suppress voluntary movement.

Table 1

Patients characteristics

\begin{tabular}{llllll}
\hline \hline & rigidity score (left) & rigidity score (right) & H-Y stage* & Duration of disease [yrs] & age [yrs] \\
\hline mean(SD) & $1.0(0.9)$ & $1.3(0.7)$ & $2.2(0.5)$ & $4.0(3.2)$ & $69.1(9.6)$ \\
\hline range & $0 \sim 2.5$ & $0 \sim 2$ & $1 \sim 3$ & $0.5 \sim 6$ & $52 \sim 86$ \\
\hline Note: The Hoehn and Yahr stage is a commonly used system for describing how the symptoms of Parkinson's disease progress.
\end{tabular}

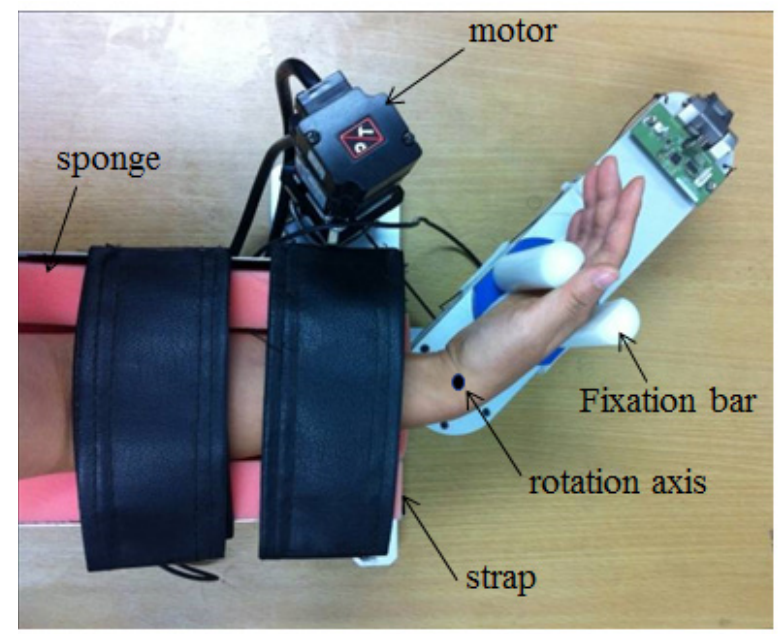

Fig. 1. Motorized passive movement device. 
The passive movement pattern consisted of 1) flexion from $30^{\circ}$ extension to $30^{\circ}$ flexion, 2) hold at $30^{\circ}$ flexion for $1 \mathrm{~s}$, extension from $30^{\circ}$ flexion to $30^{\circ}$ extension, 4) hold at $30^{\circ}$ extension for $1 \mathrm{~s}$. The pattern was repeated four times. We set the range of movement as $60 \mathrm{deg}$, which is quite reduced from the range of motion in normal subjects is $150 \mathrm{deg}$ (flexion $80 \mathrm{deg}$ to extension $70 \mathrm{deg}$ ). All patients could move their wrist in the predetermined range. No patient showed joint contracture and none of them complained of pain in this range. Both the right and left wrists were tested and the same movement pattern was applied irrespective of the left and right side for the convenience in the analysis. The angular velocity during movement was set to $30 \%$ s and $50 \%$ s.

Electromyogram (EMG) was measured throughout the experiment using wireless surface electrodes (Trigno wireless system, Delsys, MA). The reliability and measurement error of forearm surface EMG have been well documented in the literature [11], where the surface EMG was shown to have excellent intra-session reliability (intraclass correlation coefficient of 0.90 ) and low standard error of measurement. The target muscles were flexors and extensors involved in wrist joint movement, i.e., Flexor Carpi Radialis (FCR), Flexor Carpi Ulnaris (FCU), Extensor Carpi Radialis (ECR) and Extensor Carpi Ulnaris (ECU). We selected these muscles, because they were reported to be major muscles of wrist movement and to show shortening reaction as well as stretch reflex $[3,9,12]$. Also, investigation of muscles identical to those in the literature would render direct comparison with the previous results and arguments. Before attaching electrodes, the skin was cleaned with alcohol pads.

\subsection{Analyses and statistics}

Figure 2 shows the flowchart for the calculation of MSSS EMG ratio. EMG signals (four channels) were collected using a LabVIEW DAQ board. Acquired EMG signal was full wave rectified and the envelope was extracted by using Butterworth low pass filter (order: 4, Fc: $2.5 \mathrm{~Hz}$ ). The EMG of each muscle was divided into stretching phase and shortening phase. The root mean square (RMS) of the

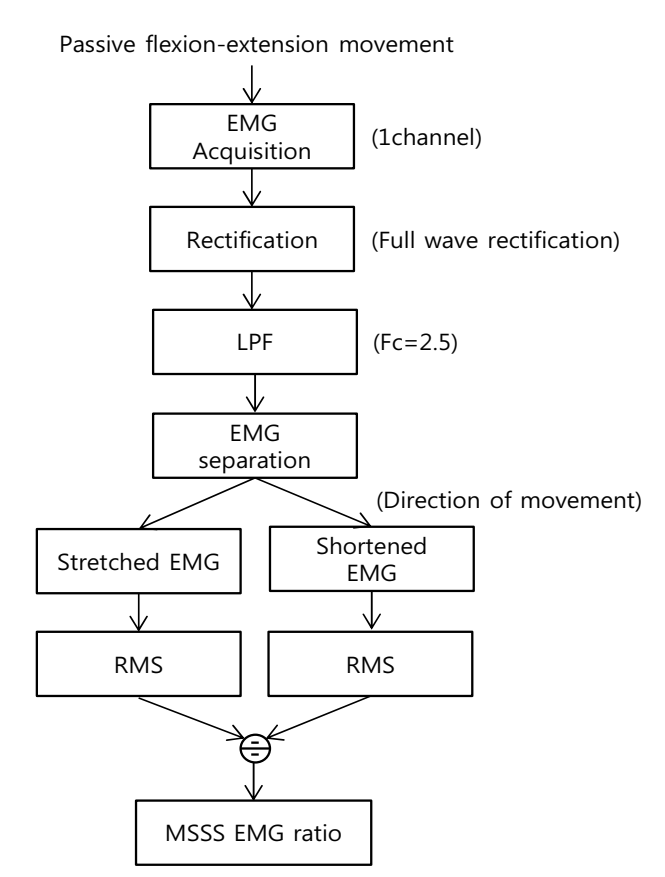

Fig. 2. Flow chart describing calculation of MSSS EMG ratio. 
EMG envelope was calculated for each phase. RMS EMG during stretching was divided by RMS EMG during shortening to derive MSSS EMG ratio.

Some of the data did not satisfy the equal-variance criteria needed for ANOVA, which made us to use non-parametric tests. Kruskal-Wallis test was used to compare MSSS EMG ratios among different rigidity scores, for each muscle. Here, the rigidity score is a nominal scale, and normal subjects were marked as $\mathrm{N}$ and patients were marked by their rigidity score ( 0 to 4$)$ rated by clinicians. KruskalWallis test was also used for the comparison of RMS EMG among subject groups, muscles, and phases.

\section{Result}

Figure 3 shows typical EMG activities during passive movement in patients with Parkinson's disease. Prominent EMG activities are marked by boxes (during stretch by blue and during shortening
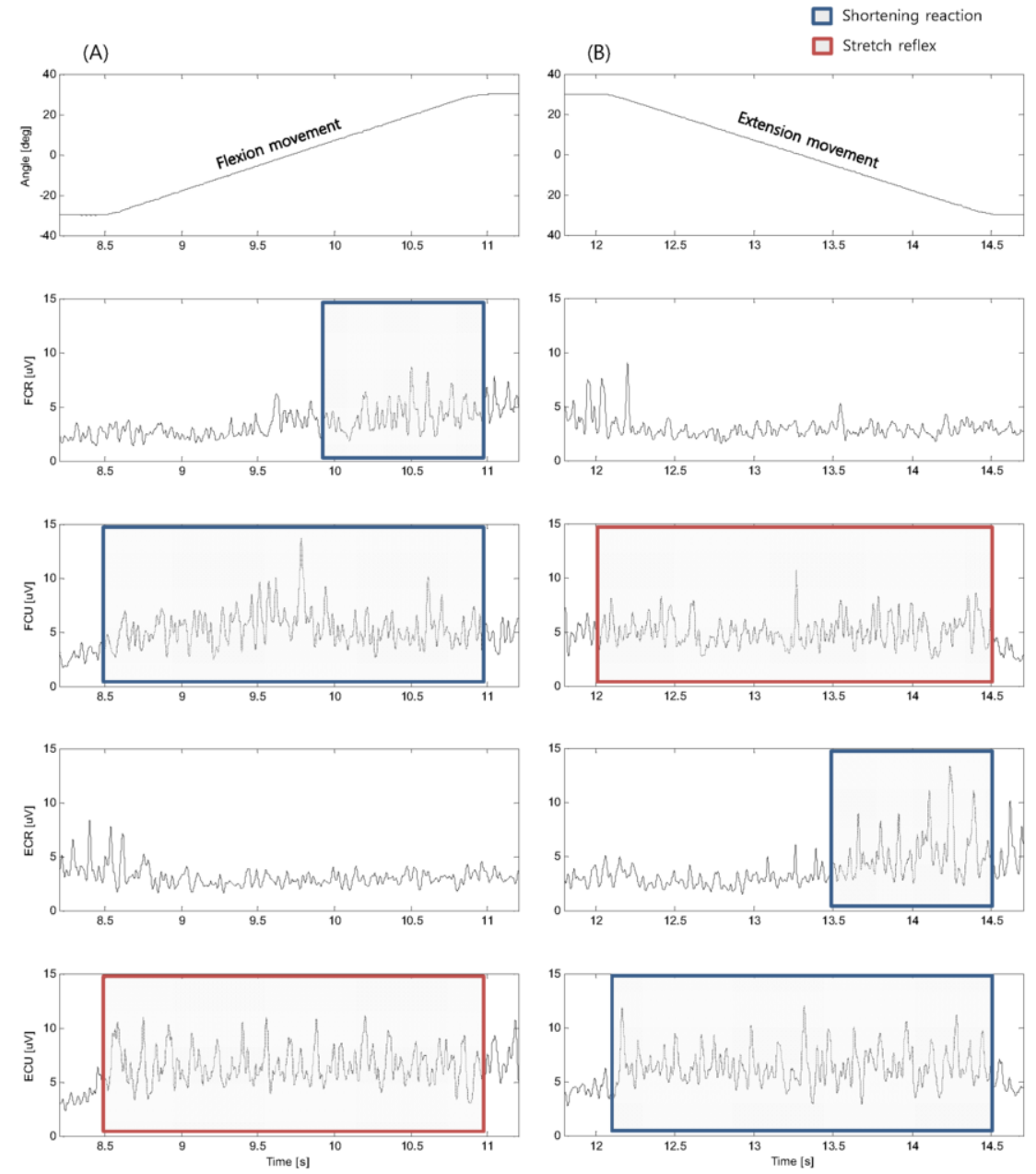

Fig. 3. EMG activities during passive flexion movement (A) and passive extension movement (B) in patients with Parkinson's disease. 


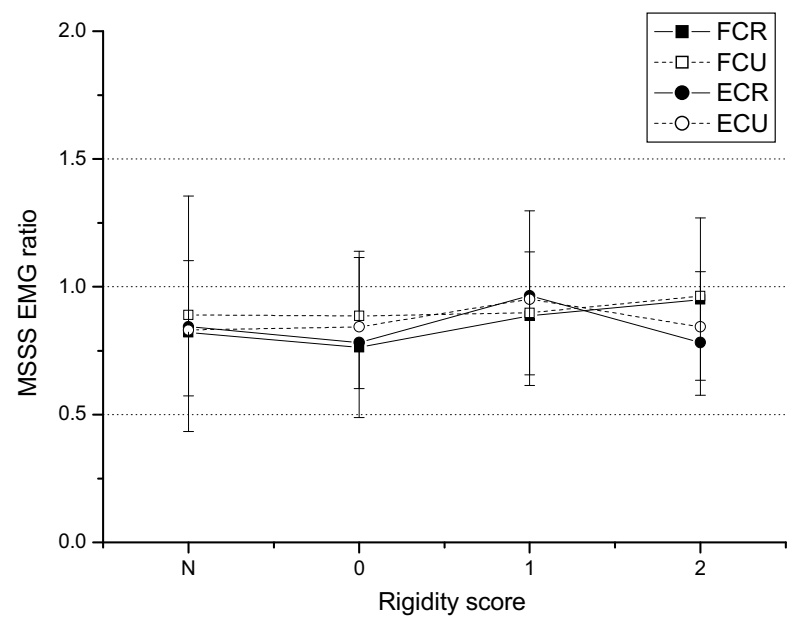

Fig. 4. Muscle-specific STR/SHR ratio in each muscle ( $\mathrm{N}$ in the horizontal axis indicates normal subjects group).

Table 2

Difference in MSSS EMG ratio among different rigidity scores

\begin{tabular}{llll}
\hline \hline muscle & & & \\
\hline FCR & FCU & ECR & ECU \\
\hline 0.058 & 0.332 & 0.091 & 0.623 \\
\hline & Note: p-value from Kruskal-Wallis test is shown in the table.
\end{tabular}

by red). This figure shows a tendency that shortening reaction is prominent in all four muscles but the stretch reflex is prominent only in FCU and ECU.

Figure 4 shows the MSSS EMG ratio with respect to the rigidity scores ( $\mathrm{N}$ indicates normal subjects), where the mean MSSS EMG ratio was always lower than one in all muscles. Table 2 shows the statistical analysis result. MSSS EMG ratio was not different among different rigidity scores in all muscles $(\mathrm{p}>0.05)$.

Figure 5 shows RMS EMG with respect subject groups, muscles, and phases. In FUC and ECR,

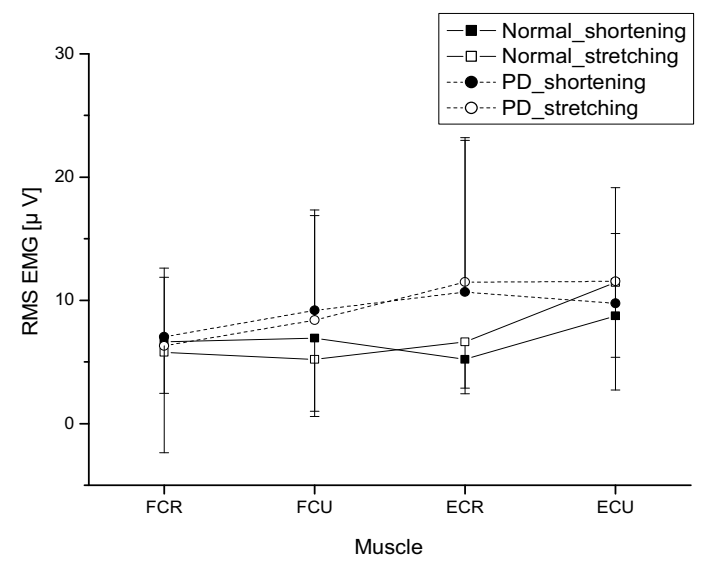

Fig. 5. RMS EMG in each group and phase. 
patients tend to show greater RMS EMG than normal subjects, irrespective of the phase. Table 3 shows the effect of group, phase, and muscle on RMS EMG. RMS EMG was significantly greater in patients compared to normal $(\mathrm{p}<0.01)$ and different among muscles. Post-hoc comparison of RMS EMG between subject groups was performed for each muscle as in Figure 6. In FCU and ECR muscles, patients showed significantly greater RMS EMG compared to normal subjects.

\section{Discussion}

The purpose of this study was to investigate the validity of suggestion of Xia et al. by using MSSS EMG ratio. In patient with PD, MSSS EMG ratio should be greater than one (at least in one muscles) to generate continuous torque resistance during passive movement if the antagonistic torques are independent and the EMG is proportional to torque. But MSSS EMG ratios were smaller than one $(0.77 \sim 0.97)$ in all muscles and all severities of rigidity (Figure 4 and Table 2). This result indicates that a greater mean activation of muscle during passive shortening than during passive stretching is common phenomenon for all muscles and also for all patients with Parkinson's disease. It is noted that the same tendency was shown even for normal subjects. Consequently, EMG ratio cannot explain the parkinsonian rigidity and denies Xia et al.'s argument that the torque resistance in rigidity is the summation of the independent contributions of stretch-reflex minus shortening reaction.

As for alternative mechanism, it is possible that the parkinsonian rigidity is generated by passive mechanical resistance of the muscle-joint system enhanced in patients. Patient with PD reduce reciprocal inhibition that control antagonist muscle activations [13-16]. The reduction of reciprocal inhibition would increase antagonistic co-contraction and make inefficient contraction of muscle.

Actually, we have confirmed that co-contraction is greater in wrist joint in PD group than normal

Table 3

Effect of group, phase, and muscle on RMS EMG

\begin{tabular}{lcc}
\hline \hline group & phase & muscle \\
\hline $\mathbf{0 . 0 0 0}$ & 0.565 & $\mathbf{0 . 0 0 0}$ \\
\hline \multicolumn{2}{c}{ Note: p-value from Kruskal-Wallis test is shown in the table. }
\end{tabular}

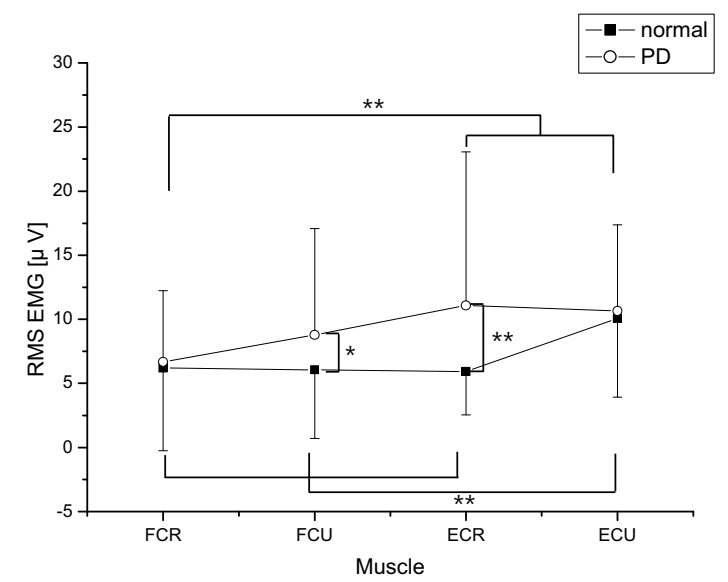

Fig. 6. Group comparison of RMS EMG (post-hoc test for group effect). 
group [10]. Also in this study, the mean EMG intensity of half of the associated muscles was much higher in patients than in normal subjects and it was consistent for both stretching and shortening phases ( $<<0.05$, Table 3 and Figure 6 ) This suggests that the enhanced muscle activations of both sides of antagonistic muscles are associated with the rigidity. The enhanced co-contraction was shown to increase mechanical resistance[17], which can fairly contribute to torque resistance in rigidity. It was also shown that the intrinsic mechanical properties muscle-joint system are altered in patients with PD [18], which can also affect rigidity. Park et al. showed that viscoelasticity represents rigidity better than the mechanical work and impulse [19]. Therefore, it is highly feasible that the rigidity is associated with the alteration in passive mechanical resistance affected by the enhanced antagonistic muscle activation and the altered intrinsic mechanical property.

The limitation of this study includes that EMG was measured at the surface and the torque resistance by rigidity was small compared to maximum voluntary contraction, where the standard error of measurement would be high (the normalized standard error of forearm surface EMG was shown to be $23-45 \%$ at low grip forces). To enhance the signal to noise ratio of EMG, needle electrodes could be used in the further study. Another interesting study would be the investigation of the rigidity mechanism by using a musculoskeletal system model and through simulation of passive movement and torque resistance with the measured EMG as input. In this way, the role and mechanism of antagonistic contractions on the rigidity could be manifested more in detail.

The conclusion of this study is that parkinsonian rigidity cannot be explained by independent contributions of resisting and assisting muscle torques, instead, it is closely associated to the enhanced co-activations of antagonistic muscle pairs. Accordingly, antagonistic co-contractions should be further investigated as a medium phase cause of parkinsonian rigidity.

\section{Acknowledgment}

This paper was supported by Konkuk University in 2015.

\section{References}

[1] A. Samii, J.G. Nutt and B.R. Ransom, Parkinson's disease, Lancet 363 (2004), 1783-1793.

[2] R. Xia, J. Sun and A.J. Threlkeld, Analysis of interactive effect of stretch reflex and shortening reaction on rigidity in Parkinson's disease, Clinical Neurophysiology 120 (2009), 1400-1407.

[3] T. Endo, R. Okuno, M. Yokoe, K. Akazawa and S. Sakoda, A novel method for systematic analysis of rigidity in Parkinson's disease, Movement Disorders 24 (2009), 2218-2224.

[4] J. Levin, S. Krafczyk, P. Valkovic, T. Eggert, J. Claassen and K. Botzel, Objective measurement of muscle rigidity in Parkinsonian patients treated with subthalamic stimulation, Movement Disorders 24 (2009), 57-63.

[5] M.B. Shapiro, D.E. Vaillancourt, M.M. Sturman, L.V. Metman, R.A. Bakay and D.M. Corcos, Effects of STN DBS on rigidity in Parkinson's disease, IEEE Transactions on Neural Systems and Rehabilitation Engineering 15 (2007), 173181.

[6] C.J. Andrews, D. Burke and J.W. Lance, The response to muscle stretch and shortening in Parkinsonian rigidity, Brain 95 (1972), 795-812.

[7] R.W. Angel and P.A. Lewitt, Unloading and shortening reactions in Parkinson's disease, Journal of Neurology Neurosurgery Psychiatry 41 (1978), 919-923.

[8] P.B. Matthews, F.W. Cody, H.C. Richardson and N. MacDermott, Observations on the reflex effects seen in Parkinson's disease on terminating a period of tendon vibration, Journal of Neurology Neurosurgery Psychiatry 53 (1990), 215-219.

[9] R. Xia and W.Z. Rymer, The role of shortening reaction in mediating rigidity in Parkinson's disease, Experimental Brain Research 156 (2004), 524-528.

[10] Y. Kwon, J.W. Kim, Y. Ho, H.M. Jeon, M.J. Bang, G.M. Eom and S.B. Koh, Analysis of antagonistic co-contractions 
with motorized passive movement device in patients with Parkinson's disease, Bio-medical Materials and Engeering 24 (2014), 2291-2297.

[11] A. Hashemi Oskouei, M.G. Paulin and A.B. Carman, Intra-session and inter-day reliability of forearm surface EMG during varying hand grip forces, Journal of Electromyography and Kinesiology 23 (2013), 216-222.

[12] R. Xia, D. Powell, W.Z. Rymer, N. Hanson, X. Fang and A.J. Threlkeld, Differentiation between the contributions of shortening reaction and stretch-induced inhibition to rigidity in Parkinson's disease, Experimental Brain Research 209 (2011), 609-618.

[13] A. Hayashi, Y. Kagamihara, Y. Nakajima, H. Narabayashi, Y. Okuma and R. Tanaka, Disorder in reciprocal innervation upon initiation of voluntary movement in patients with Parkinson's disease, Experimental Brain Research 70 (1988), 437-440.

[14] S. Lelli, M. Panizza and M. Hallett, Spinal cord inhibitory mechanisms in Parkinson's disease, Neurology 41 (1991), 553-556.

[15] S. Meunier, R. Katz and M. Simonetta-Moreau, Central nervous system lesions and segmental activity, Advances in Experimental Medicine and Biology 508 (2002), 309-313.

[16] C.H. Tsai, R.S. Chen and C.S. Lu, Reciprocal inhibition in Parkinson's disease, Acta Neurologica Scandinavica 95 (1997), 13-18.

[17] Y. Lee and J.A. Ashton-Miller, The effects of gender, level of co-contraction, and initial angle on elbow extensor muscle stiffness and damping under a step increase in elbow flexion moment, Annals of Biomedical Engineering 39 (2011), 2542-2549.

[18] V. Dietz, J. Quintern and W. Berger, Electrophysiological studies of gait in spasticity and rigidity, Evidence that altered mechanical properties of muscle contribute to hypertonia, Brain 104 (1981), 431-449.

[19] B.K. Park, Y. Kwon, J.W. Kim, J.H. Lee, G.M. Eom, S.B. Koh, J.H. Jun and J. Hong, Analysis of viscoelastic properties of wrist joint for quantification of parkinsonian rigidity, IEEE Transactions on Neural Systems and Rehabilitation Engineering 19 (2011), 167-176. 